\title{
Fungal keratitis after descemet stripping automated endothelial keratoplasty
}

\author{
Ioannis Mallias, Panagiota Mylova, Anastasia Tassiopoulou \\ Laser Plus Eye, Nea Smyrni, Athens, Greece
}

\begin{abstract}
This is a case report discussing the case of a patient with fungal infection after undergoing a descemet stripping automated endothelial keratoplasty (DSAEK). The pathogenesis and possible treatment options of fungal keratitis are discussed. Also, the precautions that should be taken in order to prevent fungal keratitis in patients undergoing DSAEK are referred to.
\end{abstract}

KEY WORDS: DSAEK, fungal keratitis, Descemet stripping automated endothelial keratoplasty

Ophthalmol J 2017; Vol. 2, No. 4, 105-109

\section{INTRODUCTION}

Fungal keratitis is an infection of the cornea caused by any of the multiple pathologic fungi capable of contaminating human beings. The progression $[1,2]$ is typically slow and it should be differentiated from other types of corneal infections, especially from bacterial ones, because they present with similar symptoms and clinical image. Some of the fungi ${ }^{1}$ that can affect the cornea are Candida spp., Aspergillus spp., Fusarium spp., Cladosporium spp., Curvularia, and Rhizopus.

Some of the risk factors [3-6] for a fungal keratitis outbreak are: trauma, ocular surface disease, ocular surgery, and topical steroid use. Also, in warmer and more humid environments, fungal keratitis is more likely to manifest. Fungal keratitis $[4,5]$ can also be associated with contact lens misuse. The infection starts [5] when the epithelial integrity is broken (trauma, surgery, ocular surface disease) and the fungus gains access into the corneal tissue. The fungus then releases proteolytic enzymes, toxins, and fungal antigens that result in corneal tissue necrosis and damage to the corneal architecture, compromising in this way the integrity and function of the entire eye.
The early diagnosis [6] of a fungal keratitis plays an essential role in the final outcome of the infected eye. Corneal ulcers $[6,7]$ that are unresponsive to broad-spectrum antibiotics, the presence of satellite lesions, and scanty secretions are some signs that should raise suspicion for the possibility of a fungal infection. Some of the symptoms of fungal keratitis are blurry vision, tearing, foreign body sensation, redness, photophobia, pain, and secretions. On the slit lamp [7] examination, the size and depth of the lesion should be assessed. If vitreous reaction is observed, then this is a sign of intraocular spread of the disease. With some kinds of fungi (filamentary fungi), the corneal lesion might have a white/greyish infiltrate with feathery borders. There might also be satellite lesions with hypopyon and conjunctival injection as well as purulent secretions. Other types [7] of fungi (yeast) cause plaque-like and a little more defined corneal ulcers, similar to bacterial infections. The above symptoms, combined with a history of trauma, especially from vegetable matter, strongly suggest the possibility of a fungal infection. In order to confirm the suspicion of fungal keratitis, a sample should be taken from deep inside the lesion and sent for culture and polymerase chain reaction (PCR) examination. 
The management of fungal keratitis consists of the use of topical and/or systemic anti-fungal medication depending on the severity of the case. In severe cases surgical treatment $[8,9]$ may be needed (conjunctival flap, therapeutic lamellar, or penetrating keratoplasty). The difficulty in treating fungal keratitis is mostly because of the delay of diagnosis. Anti-fungal medication [10-12] available for ocular therapy (mainly natamycin 5\%) has limited penetrating ability into the cornea, which makes the effect of the drug a little slow. In severe cases, intravenous medication may be preferred. Voriconazole [10-12] orally or injected into the corneal stroma can be used, as well as oral posaconazole, which is a new-generation triazole. Unfortunately, the prognosis of fungal keratitis is not very good. Severe visual acuity loss occurs in $26-63 \%$ of the patients, $15-20 \%$ need evisceration, and $31-38 \%$ of the patients proceed to penetrating keratoplasty $[2,4]$.

Patients diagnosed with fungal keratitis should be monitored daily, until a sign of improvement occurs. Corneal healing might take weeks or months. If, despite all the efforts for conservative treatment, a surgical approach is inevitable, the antimycotic medication [10-12] (topical and oral) should continue for at least two weeks postoperatively.

\section{CASE REPORT}

A female, 72 years old, visited the clinic with a severe infection on her left eye. Her eyelid was swollen, the eye was red and painful, and visual acuity was finger counting (FC) at $30 \mathrm{~cm}$ distance. The patient had undergone cataract surgery some years earlier. The patient had a Descemet Stripping
Automated Endothelial Keratoplasty (DSAEK) due to Fuchs' dystrophy that had deteriorated after cataract surgery, one month ago. The patient was on natamycin $5 \%$ five times per day, on topical voriconazole every two hours, and fluorometholone three times daily.

On a slit lamp examination performed by Dr Mallias, a corneal ulcer was observed with whitish infiltrates, and the epithelium was not intact (Fig. 1).

There was a strong clinical suspicion that the cornea was infected by atypical mycobacteria. This was the reason why we performed PCR examination both for fungi and atypical mycobacteria. Scrapings were taken from deep inside the ulcer and sent for a Polymerase Chain Reaction (PCR) examination in order to identify the cause of infection. The patient was instructed to come back for follow up examination two days later, along with the PCR results. The patient was instructed to discontinue steroid drops because it might impair the host cornea natural immune reaction. Because there was a high clinical suspicion for atypical mycobacteria, the patient was instructed to instil moxifloxacin (Vigamox) every hour and cyclopentolate hydrochloride two times per day in order to reduce ciliary spasm. The results of PCR were negative for atypical mycobacteria and positive for fungi.

The patient came for a follow-up visit, and the clinical image of the corneal ulcer was better (Fig. 2). It seemed like the discontinuation of steroid drops along with the initiation of moxifloxacin improved the situation of the infected cornea. Her visual acuity was still FC at $30 \mathrm{~cm}$ and she was still in pain.

The PCR results were positive for a fungal infection, so the treatment was altered accordingly. Moxifloxacin and natamycin 5\% were prescribed every

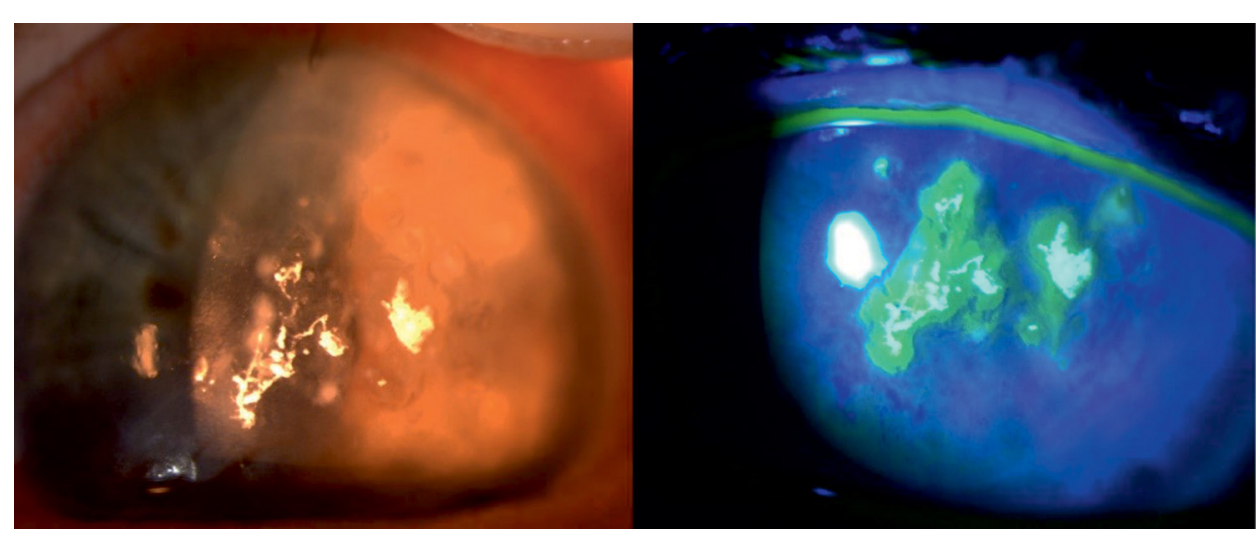

FIGURE 1. Slit lamp picture from the first visit of the patient. A corneal ulcer is observed with white infiltrates. Also, there is an epithelial defect 


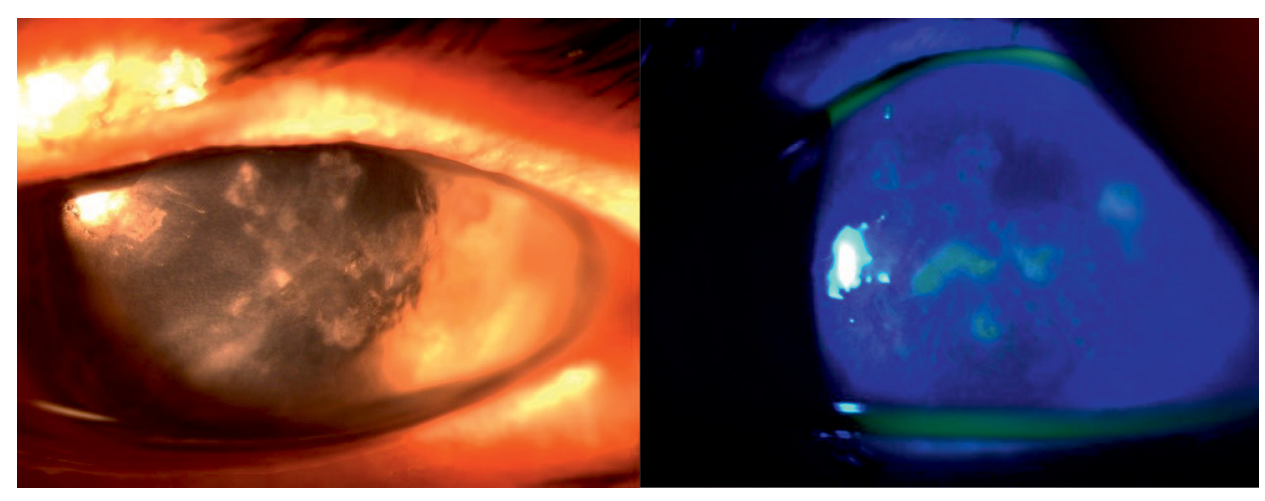

FIGURE 2. Slit lamp photo of the left eye of the patient 3 days after the change of treatment. The epithelium is significantly improved since the previous visit

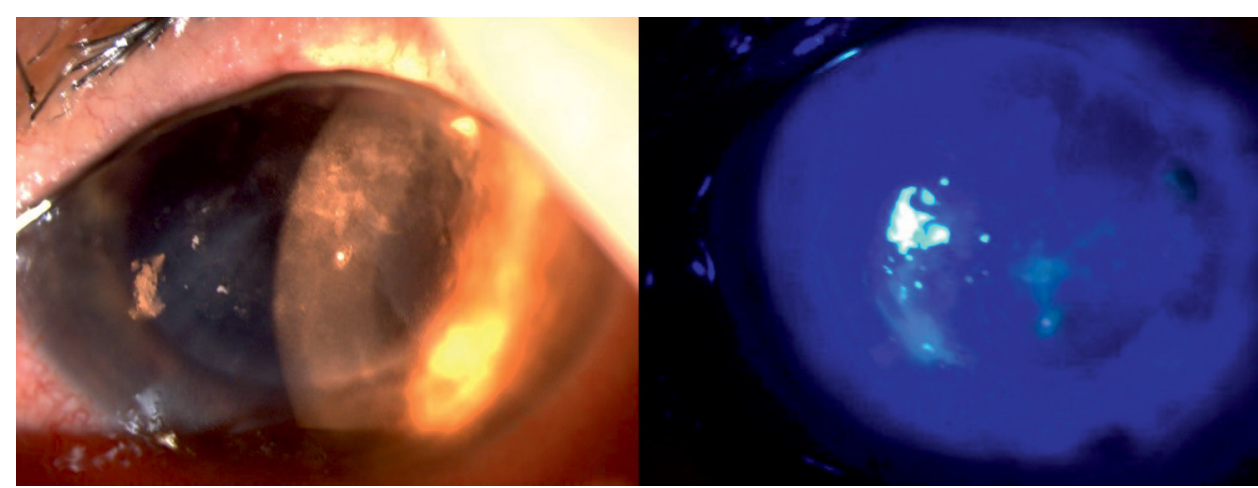

FIGURE 3. Slit lamp photo of the left eye of the patient after the change of treatment, adding anti-fungal medication

hour and cyclopentolate hydrochloride two times daily. The patient was asked to return for a follow up examination four days later.

The patient came for the scheduled follow-up. The pain on her left eye was slightly better, but the lid was still swollen and her eye was still very irritated, probably due to irritation caused by the antifungal medication. However, the clinical image of the corneal ulcer was improved (Fig. 3). The epithelium was almost healed and there was still inflammation, but the whitish infiltrates had started to fade, which is a sign of the eradication of the fungal infection.

The treatment was again slightly altered, adding oral voriconazole $(200 \mathrm{mg})$ two times daily. The patient was asked to come back in 10 days.

When the patient came back 10 days later, the clinical image of the cornea was improved and her visual acuity was improved as well (FC at $1.5 \mathrm{~m}$ ). On the slit lamp image (Fig. 4) the improvement is obvious because the haziness of the cornea is significantly improved.

The patient was then advised to come back 10 days later.

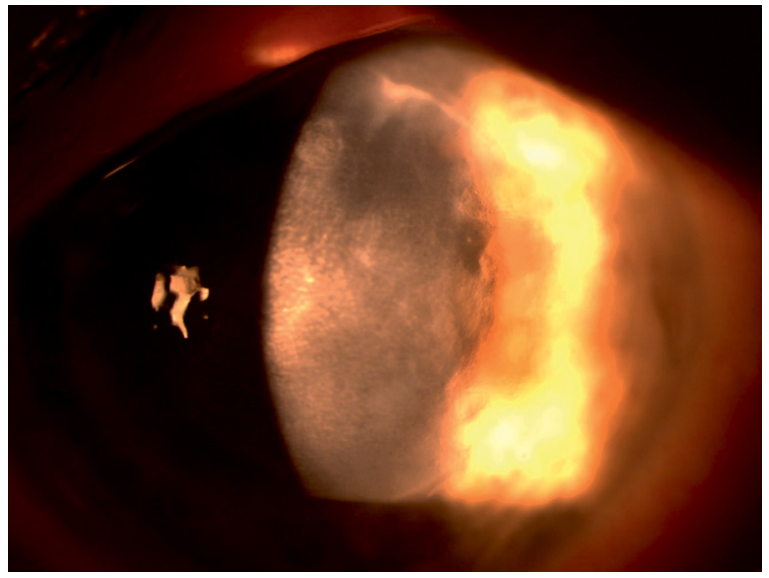

FIGURE 4. Significantly improved image of the infected eye of the patient, 15 days after treatment initiation

On the patient's fifth visit, almost a month after treatment initiation, the cornea was significantly improved. The infiltrates were completely resolved, which means the fungus was completely eradicated and the epithelium was completely healed. A mild haze was observed where the ulcer used to be (Fig. 5). 


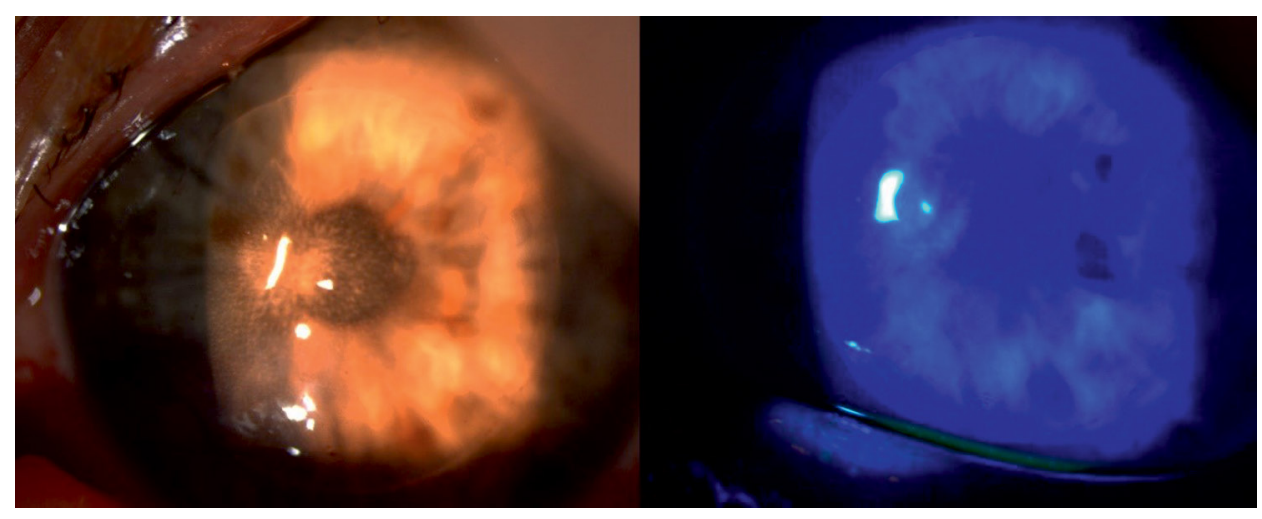

FIGURE 5. Slit lamp photo from the fifth visit of the patient, 25 days after treatment initiation.

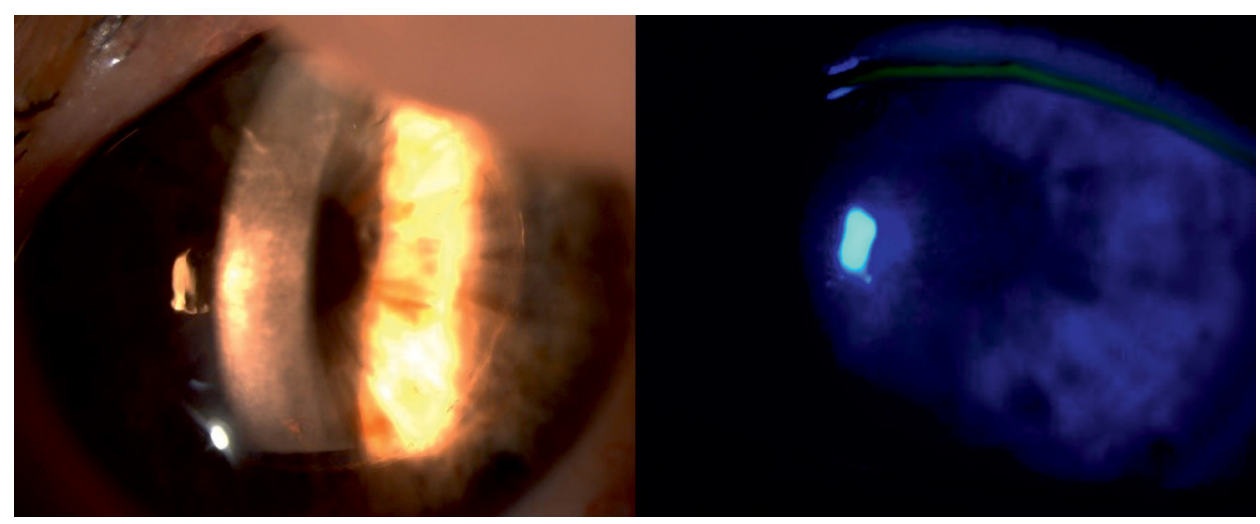

FIGURE 6. Slit lamp photo showing the improvement of the cornea, a full month after treatment initiation

The pain was eradicated, and the patient only felt a little discomfort when instilling the drops.

The visual acuity remained FC at $1.5 \mathrm{~m}$, as in the last visit. The treatment was kept the same and the patient was asked to come for a follow-up 10 days later.

After 10 days, the clinical image on the slit lamp was even more improved (Fig. 6). The haze had improved and the visual acuity was 20/200.

\section{DISCUSSION}

As described in the literature, fungal infection after a DSAEK surgery is quite a common complication [13]. There are several studies [14, 15] indicating that it is possible for a patient undergoing DSAEK surgery to present with fungal interface keratitis due to pre-existing donor infection. For that reason, these studies suggest that donor corneal rim cultures should be prophylactically performed in all cases, and confocal microscopy on cases with new interface opacities in the early postoperative period, in order to start the tissue recipient on antifun- gal therapy as soon as possible. Another study [16] indicates that the fungi can penetrate an eye that has undergone a second DSAEK (due to primary graft failure) through the corneal venting incisions that were reopened for the second surgery.

Also, there are studies [17] that present the management of late onset fungal keratitis in patients that have undergone DSAEK. The two patients in this study did not want to undergo further surgery in order to deal with the fungal infection, and topical and oral therapy had already failed. These patients received three to four intrastromal injections with antifungal medication, and it was proven to be effective in treating late-onset fugal keratitis.

In the case presented above, it could not be assertained if the fungal infection was due to previous donor infection or the recipient patient was contaminated during or after the surgery. The symptoms started about 20 days after the DSAEK surgery, and the patient informed us that she was occupied with agricultural activities during this time, which makes it possible that the infection started postoperatively. However, we have no knowledge if donor corneal 
rim cultures were made, because we did not perform the operation.

Fortunately, the patient responded very well to the cortisone discontinuation and to the conservative oral and topical treatment, without any rejection outbreaks. The cornea cleared from the ulcer and the haze almost completely and the visual acuity improved as well. If despite our efforts, oral and topical treatment had been inadequate and the infection had spread to the anterior chamber, our first choice would have been intrastromal injections with antifungal medication. If the injections had not provided any positive outcome, then the solution would have to have been surgical, with a penetrating keratoplasty.

The take home message is to discontinue steroid drops until the complete eradication of the micro-organism. Our experience is that steroid drops should not be re-introduced, at least for one month.

\section{REFERENCES}

1. O'Day DM. Fungal keratitis. In: Leibowitz HM, Waring GO. ed. Corneal Disorders: Clinical Diagnosis and Management. Saunders, Philadelphia 1998: 711-718.

2. Ibrahim M, Vanini $R$, Ibrahim $F$, et al. Epidemiologic aspects and clinical outcome of fungal keratitis in Southeastern Brazil. European Journal of Ophthalmology. 2018; 19(3): 355-361, doi: 10.1177/112067210901900305.

3. Ritterband DC, Seedor JA, Shah MK, et al. Fungal keratitis at the new york eye and ear infirmary. Cornea. 2006; 25(3): 264-267, doi: 10.1097/01.ico.0000177423.77648.8d, indexed in Pubmed: 16633023.

4. Rondeau N, Bourcier T, Chaumeil C, et al. Fungal keratitis at the Centre Hospitalier National d'Ophtalmologie des Quinze-Vingts: retrospective study of 19 cases. J Fr Ophtalmol. 2002; 25(9): 890-896.

5. AAO, Focal Points: Diagnosis and Management of Fungal Keratitis, Module. ; 6: 2002.
6. Bron AJ, Seal DV, Hay J. Ocular infection: Investigation and treatment in practice. Mosby, St Louis 1998.

7. O'Brien T. Therapy of ocular fungal infections. Ophthalmol Clin North Am. . 1999; 12(1): 33-50, doi: 10.1016/s0896-1549(05)70147-4.

8. Kepez Yildiz B, Hasanreisoglu M, Aktas Z, et al. Fungal keratitis secondary to Scedosporium apiospermum infection and successful treatment with surgical and medical intervention. Int Ophthalmol. 2014; 34(2): 305-308, doi: 10.1007/s10792-013-9777-8, indexed in Pubmed: 23612853.

9. Imtirat A, Levy J, Lifshitz T. Treatment of fungal keratitis by penetrating keratoplasty. Harefuah. 2010; 149(3): 166-9, 194, indexed in Pubmed: 20684168.

10. Prajna NV, Krishnan T, Rajaraman R, et al. Mycotic Ulcer Treatment Trial II Group, Mycotic Ulcer Treatment Trial Group. The mycotic ulcer treatment trial: a randomized trial comparing natamycin vs voriconazole. JAMA Ophthalmol. 2013; 131(4): 422-429, indexed in Pubmed: 23710492.

11. Prakash G, Sharma N, Goel M, et al. Evaluation of intrastromal injection of voriconazole as a therapeutic adjunctive for the management of deep recalcitrant fungal keratitis. Am J Ophthalmol. 2008; 146(1): 56-59, doi: 10.1016/j.ajo.2008.02.023, indexed in Pubmed: 18436173.

12. Bunya $\mathrm{V}$, Hammersmith KM, Rapuano CJ, et al. Topical and oral voriconazole in the treatment of fungal keratitis. Am J Ophthalmol. 2007; 143(1): 151-153, doi: 10.1016/.ajo.2006.07.033, indexed in Pubmed: 17188052.

13. Koenig SB, Wirostko WJ, Fish RI, et al. Candida keratitis after descemet stripping and automated endothelial keratoplasty. Cornea. 2009; 28(4): 471-473, doi: 10.1097/ICO.0b013e31818ad9bc, indexed in Pubmed: 19411972.

14. Kitzmann AS, Wagoner MD, Syed NA, et al. Donor-related Candida keratitis after descemet stripping automated endothelial keratoplasty. Cornea. 2009; 28(7): 825-828, doi: 10.1097//C0.0b013e31819140c4, indexed in Pubmed: 19574899.

15. Lee WB, Foster JB, Kozarsky AM, et al. Interface fungal keratitis after endothelial keratoplasty: a clinicopathological report. Ophthalmic Surg Lasers Imaging. 2011; 42: e44-e48, doi: 10.3928/1542887720110407-01, indexed in Pubmed: 21485974.

16. Chew ACY, Mehta JS, Li L, et al. Fungal endophthalmitis after descemet stripping automated endothelial keratoplasty - a case report. Cornea. 2010; 29(3): 346-349, doi: 10.1097/ICO.0b013e3181a9d0c0, indexed in Pubmed: 20118788.

17. Tu EY, Hou J. Intrastromal antifungal injection with secondary lamellar interface infusion for late-onset infectious keratitis after DSAEK. Cornea. 2014; 33(9): 990-993, doi: 10.1097/IC0.0000000000000192, indexed in Pubmed: 25055150. 